POS $\quad \begin{aligned} & \text { PROCEEDINGS } \\ & \text { OF SCIENCE }\end{aligned}$

\title{
Collectivity in $\mathrm{pPb}$ and $\mathrm{pp}$ collisions with the string percolation model
}

\author{
I. Bautista ${ }^{* 1,2}$, R. Alvarado ${ }^{2}$, P. Fierro ${ }^{2}$ \\ ${ }^{1}$ Cátedra-CONACYT ${ }^{2}$ Facultad de Ciencias Físico Matemáticas, Benemérita Universidad \\ Autónoma de Puebla,1152, México \\ E-mail: irais.bautista@fcfm.buap.mx
}

By using the string percolation framework we study the shear viscosity over entropy ratio in high multiplicity events in $\mathrm{pPb}$ and $\mathrm{pp}$ collisions at the current LHC energies, where evidence on collective like effects has been found recently on data. We show the comparison of the obtained values with the nucleus-nucleus collisions where the strong interacting Quark Gluon Plasma phase is formed.

38th International Conference on High Energy Physics 3-10 August 2016

Chicago, USA

${ }^{*}$ Speaker. 


\section{Introduction}

The String percolation model (SPM) have described successfully the collective effects on high energy Quantum Chromodynamics (QCD) matter formed at heavy ion collisions from the Relativistic Heavy Ion Collider (RHIC) to the Large Hadron Collider (LHC) energies [1][2][3]. The relationship with the QCD phase diagram can be derived in terms of the thermodynamical quantities [3]. In these manuscript we extend the results to study the signatures of collective effects which has been shown on recent results on $\mathrm{p}-\mathrm{p}$ and $\mathrm{p}-\mathrm{Pb}$ (small systems) collisions measured by the collaborations ALICE, CMS and ATLAS at LHC energies [4][5], and which origin is still not fully understand.

\section{The model}

In the string percolation model, the interaction between colliding nuclei is effectively represented by the formation of extended color flux tubes namely strings, which are stretching among the partons of the projectile and target. The formed strings carry a fraction of the parton momentum, and have a extended color field between the color charges at their ends.

If we project the strings in the impact parameter plane, their projection can be seen as small discs of radius $r_{0}$, where 2 dimensional continuos percolation theory can be applied. The number of strings will grow (with energy, atomic number and centrality) causing overlapping and forming clusters which for a critical value will start to percolate. The strings will produce particles by string breaking and pair creation $(q \bar{q}$ and $q q-\bar{q} q)$ with the Schwinger Mechanism, and latter they will hadronize.

The color charges summation of the clusters gives a reduction in multiplicity, an increase in the string tension of the cluster, and also a increase on the average momentum fraction of the partons $\left\langle p_{T}^{2}\right\rangle$. The transverse color string density $\zeta_{t}=\frac{\pi r_{0}^{2}}{S} N^{s}$ which is defined in terms of the number of strings $N^{S}$ and the overlapping area $S$, was found to have the critical value value of 1.15 and 1.5 depending on the nuclear profile function, homogeneous or Wood Saxon [6]. For values of $\zeta^{t}$ over the critical value, a formation long strings will appear due to string fusion.

In pp collisions the transverse string density can be written as $\zeta^{t}=\left(\frac{r_{0}}{R_{p}}\right)^{2} N^{s}$ where $r_{0} \simeq 0.25 \mathrm{fm}$ is the transverse size of a single string, $R \simeq 1 \mathrm{fm}$ is the transverse size of a proton and $N^{s}$ is the number of strings in pp collisions given by:

$$
N_{p}^{s}=2+\left(\frac{2 r_{0}}{R}\right)^{2}\left(\frac{\sqrt{s}}{m_{p}}\right)^{2 \lambda},
$$

with $m_{p}$ the proton mass and with a constant parameter $\lambda \simeq 0.201$

Another important quantity in the SPM, is the color reduction factor $F\left(\zeta^{t}\right)$, which is related with the particle and string density by:

$$
\frac{d N}{d y}=\kappa F\left(\zeta^{t}\right) N^{s}
$$


where $\kappa \sim 0.63$ is a normalization factor and $F\left(\zeta^{t}\right)=\sqrt{\frac{1-e^{-\zeta^{t}}}{\zeta^{t}}}$ is the color reduction factor that slows down the rate of increase on particle density with the energy and the number of strings.

The total multiplicity is obtained through the measurement over all the cluster configurations, considering only the contributing clusters on the central region.

\section{Results}

To determine the corresponding value of the strings density, we use the corresponding transverse identified particle spectrum, given by:

$$
\frac{1}{2 \pi N p_{T}} \frac{d^{2} N}{d \eta d p_{T}}=\frac{a\left(p_{0}\right)^{\alpha-2}}{p_{T}\left(p_{T}+p_{0}\right)^{\alpha-1}}
$$

where $a, p_{0}$ and $\alpha$ are energy dependent parameters, which values are obtained from fitting data [7] with equation (3.1) as done in reference [8], the procedure was applyied to $\mathrm{pPb}$ collisions at $5.02 \mathrm{TeV}$ and the results are shown in table 1.

$$
\begin{array}{|c|c|c|}
a & p_{0} & \alpha \\
\hline 181 \pm 27 & 3.24 \pm .53 & 10.66 \pm .71
\end{array}
$$

Table 1. Fit parameters to minimum bias transverse momentum distribution

One can correlate high multiplicity events (with string density $\zeta_{H M}$ ) and minimum bias distribution as

$$
\left.\frac{1}{N} \frac{d^{2} N_{c h}}{d \eta d p_{T}}\right|_{\eta=0}=a \frac{\left(p_{0} b\right)^{\alpha-2}}{\left(p_{T}+p_{0} b\right)^{\alpha-1}}
$$

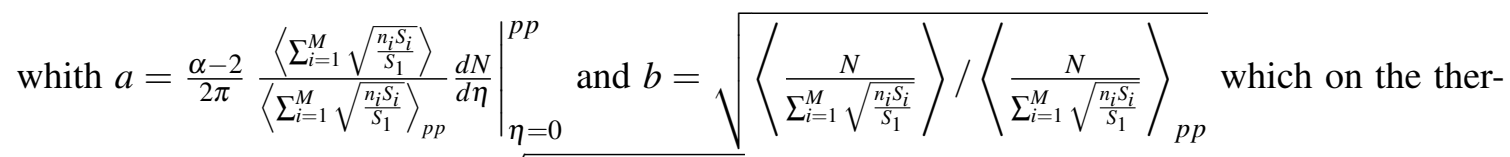
modynamic limit become $b \rightarrow \sqrt{F(\zeta) / F\left(\zeta_{H M}\right)}$, thus we have:

$$
\frac{1}{N} \frac{d^{2} N}{d \eta d p_{T}}=a \frac{\left(p_{0} \sqrt{\frac{F\left(\zeta_{p p}\right)}{F\left(\zeta_{H M}\right)}}\right)^{\alpha-2}}{\left(p_{T}+p_{0} \sqrt{\frac{F\left(\zeta_{p p}\right)}{F\left(\zeta_{H M}\right)}}\right)^{\alpha-1}}
$$

The color string density on high multiplicity events in $\mathrm{p}-\mathrm{p}$ and $\mathrm{p}-\mathrm{Pb}$ collisions was obtained by fitting the $\frac{1}{N 2 \pi p_{T}} \frac{d^{2} N}{d \eta d p_{T}}$ which expression can be derived from equation (3.3) with data from CMS and ALICE.

On figure 1 we show the fits obtained for charged pions in $\mathrm{p}-\mathrm{Pb}$ at $5.02 \mathrm{TeV}$ [7] in a similar manner as it was done for pp collisions on reference [8]. Notice that the fits are better for higher multiplicities. 


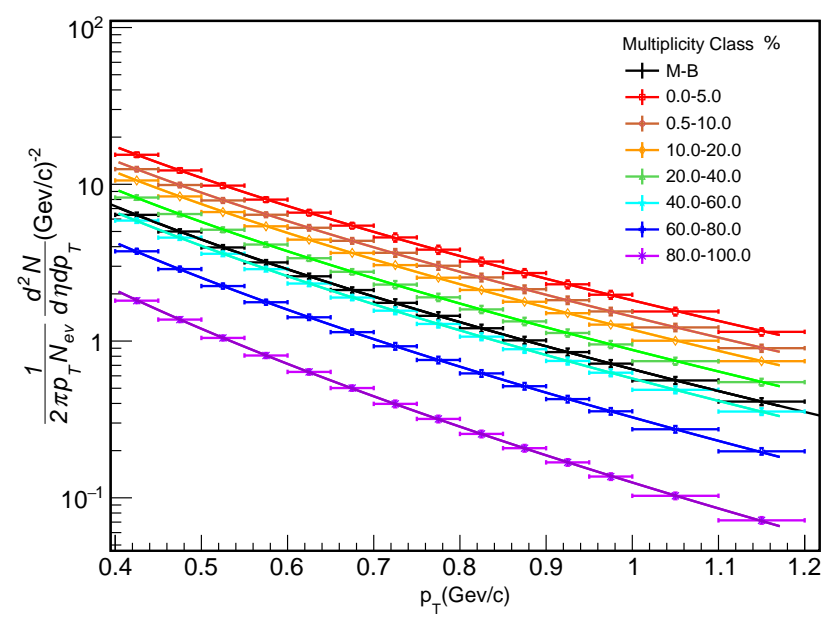

Figure 1: Fits to the transverse momentum distribution for $\mathrm{p}-\mathrm{Pb}$ at $\sqrt{s}=5.02 \mathrm{TeV}$ data taken from reference [7].

Figures 2 shown the obtained $F(\zeta)$ values for $\mathrm{pPb}$ and $\mathrm{p}-\mathrm{p}$ collisions for the corresponding energies
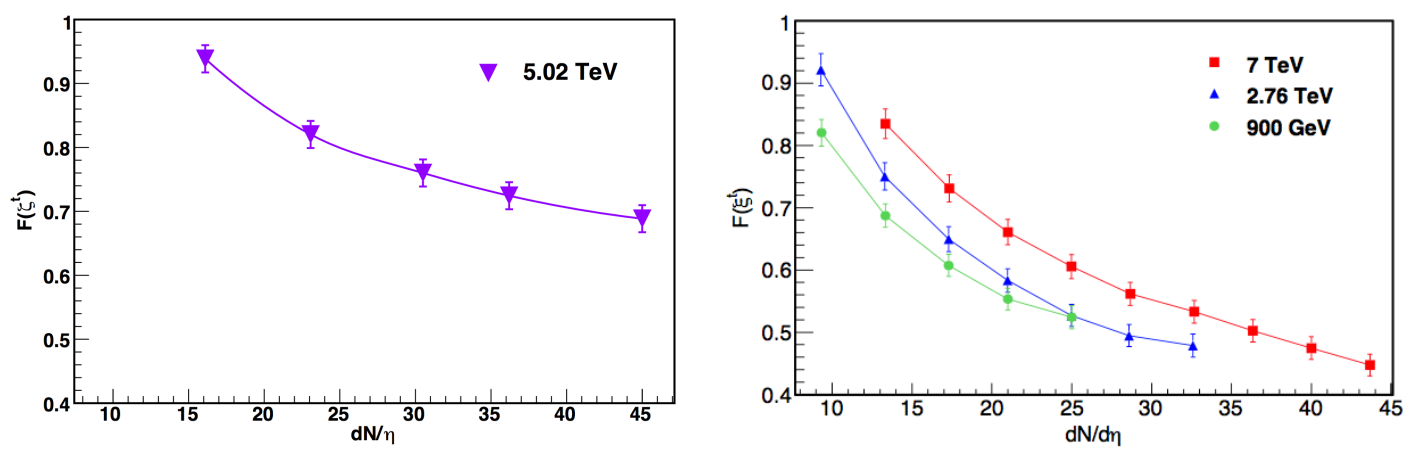

Figure 2: Obtained values of the color reduction function for $\mathrm{p}-\mathrm{Pb}$ at $\sqrt{s}=5.02 \mathrm{TeV}$ (right), and p-p collisions from .9 to $7 \mathrm{TeV}$ (left).

Moreover we consider from the Schwinger mechanism for non massive particles the transverse momentum distribution $\left(\frac{d N}{d p_{T}} \sim e^{-2} \sqrt{2 F\left(\zeta^{T}\right)} \frac{p_{T}}{\left\langle p_{T}\right\rangle_{1}}\right)$, and taking into account the mean strength of the string fluctuating around its mean value due to that the chromodynamic field is not constant $\left\langle x^{2}\right\rangle=\pi\left\langle p_{T}^{2}\right\rangle_{1} / F\left(\zeta^{t}\right)$. A no Gaussian distribution of the fluctuations of the string tension gives rise to a thermal distribution with $T\left(\zeta^{t}\right)=\sqrt{\frac{\left\langle p_{T}^{2}\right\rangle_{1}}{2 F\left(\zeta^{t}\right)}}$. As in reference [8] we consider $T_{c}=154 \pm 9 \mathrm{MeV}$ from the HotLQCD collaboration, and $\left\langle p_{T}\right\rangle_{1}=190.25 \pm 11.2 \mathrm{MeV} / \mathrm{c}$ consistent with the direct photon enhanced. By using the corresponding effective temperature for $\mathrm{p}-\mathrm{Pb}$ and $\mathrm{p}-\mathrm{p}$ collisions obtained from the thermal distribution, we study the ratio of the shear viscosity over entropy density, mean transverse momentum. Considering the relativistic kinetic theory $\eta / s \simeq \frac{T \lambda_{f p}}{5}$ with $\lambda_{f p} \sim \frac{1}{n \sigma_{t r}}$ (mean free path), $n=\frac{N_{\text {sources }}}{S_{N} L}$ (density of effectives sources per unit volume), $\sigma_{t r}=\frac{S_{N}\left(1-e^{-\zeta^{t}}\right.}{N_{\text {sources }}}$ (trans- 
verse area of the effective string) the ratio of shear viscosity over entropy is given as

$$
\frac{\eta}{S}=\frac{T L}{5\left(1-e^{-\zeta^{t}}\right)}
$$
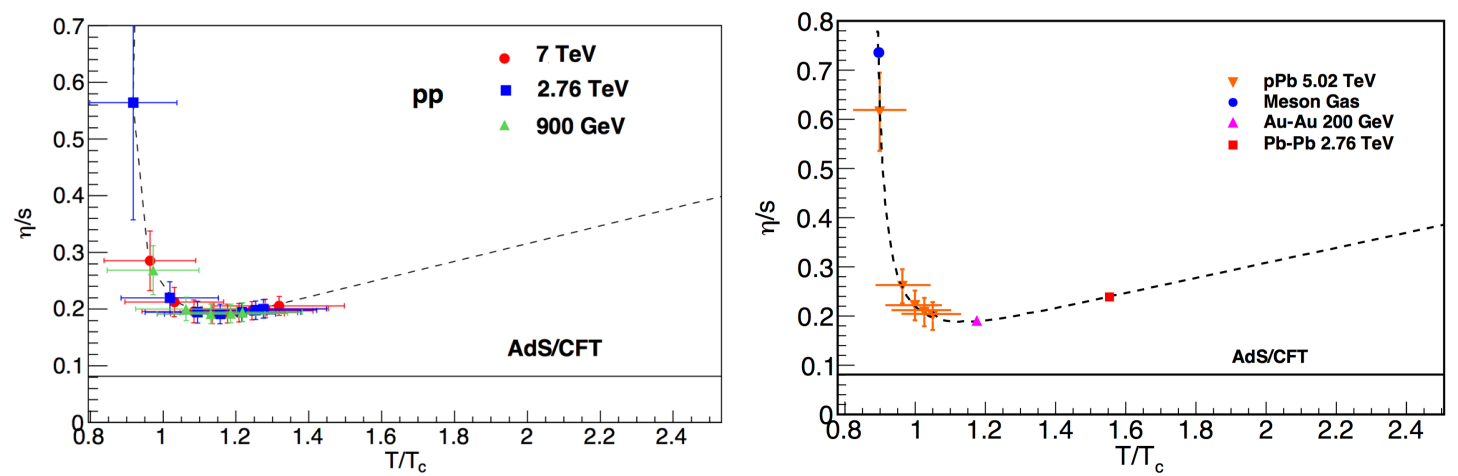

Figure 3: Shear viscosity over entropy for $\mathrm{p}-\mathrm{Pb}$ at $\sqrt{s}=5.02 \mathrm{TeV}$ (right), and for $\mathrm{p}-\mathrm{p}$ collisions from .9 to $7 \mathrm{TeV}$ (left).

\section{Conclusions}

The obtained results for the shear viscosity over entropy ratio exhibit a similar trend consistent with pp collisions shown in figure 3, but below the obtained values corresponding to $\mathrm{Au}-\mathrm{Au}$ collisions at $200 \mathrm{GeV}$ where the QGP formation has been claimed. Results from $d N / d \eta \sim 16$ are close to the Meson Gas (MG) value which indicates that below this multiplicity regime there is a clear dilute state.

\section{References}

[1] I. Bautista, C. Pajares, J. G. Milhano and J. Dias de Deus, Phys. Rev. C 86 (2012) 034909

[2] I. Bautista, J. Dias de Deus and C. Pajares, Acta Phys. Polon. Supp. 6 (2013) 165.

[3] M. A. Braun, J. Dias de Deus, A. S. Hirsch, C. Pajares, R. P. Scharenberg and B. K. Srivastava, Phys. Rept. 599 (2015) 1

[4] R. Bala, I. Bautista, J. Bielcikova and A. Ortiz, Int. J. Mod. Phys. E 25 (2016) no.07, 1642006

[5] C. Loizides, Nucl. Phys. A 956 (2016) 200 D. Stau er, A. Aharony, Introduction to percolation theory CRC Press, 1994.

[6] A. Rodrigues, R. Ugoccioni and J. Dias de Deus, Phys. Lett. B 458 (1999) 402

[7] B. B. Abelev et al. [ALICE Collaboration], Eur. Phys. J. C 74 (2014) no.9, 3054

[8] I. Bautista, A. F. TŐllez and P. Ghosh, Phys. Rev. D 92 (2015) no.7, 071504 